\title{
The study of platelet receptors using artificial lipid bilayers
}

Michael L. Dustin ${ }^{1}$ and Alice Y. Pollitt ${ }^{2}$

${ }^{1}$ Kennedy Institute of Rheumatology, University of Oxford, Roosevelt Drive, Headington, Oxford, OX3 7FY, UK

${ }^{2}$ School of Biological Sciences, University of Reading, Whiteknights, Reading, RG6 6AS, UK

Correspondence: a.pollitt@ reading.ac.uk

Running Head: artificial lipid bilayers

\begin{abstract}
Artificial lipid bilayers are powerful tools that can be used to model the interactions between platelets and membrane bound ligands. To mimic the interaction of platelets with membrane bound ligands, biotinylated lipids can be used to couple monobiotinylated recombinant ligands to the upper leaflet of an artificial lipid bilayer using streptavidin to bridge the two. Artificial lipid bilayers are generated by preparing liposomes, treating glass coverslips to make them hydrophilic and by assembling the bilayer in a specialized flow chamber. Finally platelets can be added to the flow chamber and the localisation of fluorescently labelled molecules followed using microscopy.
\end{abstract}

\section{Key words}

Artificial lipid bilayer, receptor ligand interactions, CLEC-2, Podoplanin

\section{Introduction}

Artificial lipid bilayers, also referred to as planar lipid bilayers, have made a tremendous impact in the field of immunology to study the immunological synapse formed by T, B, and natural killer cells [1-3] and more recently in the study of platelet receptors [4].

This protocol describes the preparation of artificial lipid bilayers containing dioleoyl phosphatidylcholine and biotinylated lipids. Commercially available biotinylated lipids enable the incorporation of monobiotinylated proteins and antibodies to the upper leaflet of an artificial lipid 
bilayer using streptavidin to bridge the two [5]. The lipid dioleoyl phosphatidylcholine is used to adjust the density of the biotinylated lipids and alone acts as a negative control. By adjusting the density of biotinylated lipids the density of proteins incorporated into the upper leaflet of the bilayer can be carefully controlled to mimic those of endogenously expressed proteins.

Artificial lipid bilayers can model the interaction of platelets with membrane bound ligands. One such interaction studied using this method is between the platelet receptor CLEC-2 and the platelet ligand Podoplanin, a transmembrane protein expressed on a variety of cell types [6]. Artificial lipid bilayers containing monobiotinylated recombinant podoplanin extracellular domain have enabled the dynamics of platelet podoplanin interactions to be visualised [4].

The general principles described here can also be used to produce artificial lipid bilayers containing other lipids. Suitable lipids need to have a transition temperature below room temperature to ensure that the lipids remain in their fluid state. Liposomes containing commercially available nickel chelating lipids can be used to generate artificial lipid bilayers where histidine tagged proteins can be tethered to the upper leaflet of the bilayer [5,7]. The method also opens up the possibility of generating a procoagulant surface containing phosphatidylserine lipids to investigate the formation of cell surface coagulation complexes.

\section{Materials}

Prepare all solutions using ultrapure water and analytical grade reagents. Carefully comply with local standard operating procedures when disposing of waste materials.

1. Degassed Tris Buffered Saline (TBS): Prepare $6 \times 1 \mathrm{~L}$ of $1 \mathrm{x}$ TBS in conical flasks $(25 \mathrm{mM}$ Tris, $150 \mathrm{mM} \mathrm{NaCl}, \mathrm{pH} 8$ ). Deoxygenate the buffer by bubbling through Nitrogen using a $70 \%$ ethanol treated glass diffuser for 15 minutes per flask (see Note $^{1}$ ). Once degassed, purge with argon, seal and store at $4^{\circ} \mathrm{C}$. This buffer will be used to dilute liposomes and to dialyze away detergent.

2. $10 \%$ Octyl $\beta$-D-glucopyranoside: prepare a $10 \% \mathrm{w} / \mathrm{v}$ Octyl $\beta$-D-glucopyranoside in water, filter through $0.22-\mu \mathrm{m}$ filter and kept as frozen stocks. 
3. $2 \%$ Octyl $\beta$-D-glucopyranoside: $20 \mathrm{ml}$ freshly prepared by dilution of $10 \%$ Octyl $\beta$-Dglucopyranoside in degassed $1 \mathrm{x}$ TBS. Store on ice until ready to use.

4. Piranha solution: In a fume hood and wearing appropriate personal protective equipment (see Note $^{2}$ ) prepare in a clean, $100 \mathrm{ml}$ dry Pyrex beaker placed in the centre of a Pyrex dish. The Pyrex dish will contain any spillages should they occur. Slowly add $15 \mathrm{ml}$ of $30 \% \mathrm{w} / \mathrm{w}$ hydrogen peroxide $\left(\mathrm{H}_{2} \mathrm{O}_{2}\right)$ to $35 \mathrm{ml}$ of concentrated sulphuric acid $\left(\mathrm{H}_{2} \mathrm{SO}_{4}\right)$ while stirring with a clean dry glass rod.

5. Dialysis tubing preparation: Cut $8-10 \mathrm{~cm}$ pieces of dialysis tubing, Molecular Weight cut-off: 12-14 kDa, $10 \mathrm{~mm}$ diameter. Place in beaker with ultrapure water and sterilize the tubing by microwaving until the water boils.

6. Blocking buffer: $5 \mathrm{mg} / \mathrm{ml}$ bovine serum albumin (BSA) in 1x PBS solution. Weigh $0.2 \mathrm{~g}$ of fatty acid free BSA into a $50 \mathrm{ml}$ polypropylene tube and make up to $40 \mathrm{ml}$ with 1x PBS. Place into a boiling water bath for 5 mins. Transfer on to ice to cool. Once cool pass through a $0.22-\mu \mathrm{m}$ filter and aliquot into $5 \mathrm{ml}$ volumes. Aliquots can be kept frozen and brought up to room temperature when needed.

7. $10 \mu \mathrm{g} / \mathrm{ml}$ streptavidin: $10 \mu \mathrm{g} / \mathrm{ml}$ streptavidin diluted in blocking buffer.

\section{Methods}

\section{Preparing liposomes}

These protocol notes describe the preparation of bilayers containing 1,2-dioleoyl-sn-glycero-3phosphocholine (DOPC; Avanti lipids) and biotinylated lipids (1,2-dioleoyl-sn-glycero-3phosphoethanolamine-N-(cap biotinyl)) (CAP-BIOTIN-PE; Avanti lipids). Biotinylated lipids can couple monobiotinylated proteins (see Note $^{3}$ ) to the upper leaflet of an artificial lipid bilayer. Other lipids can be prepared similarly (see Note $^{4}$ ). 


\subsection{Evaporation and lyophilisation of chloroform from lipids}

1. In a fume hood add approximately $2 \mathrm{ml}$ of high grade chloroform to a $16 \times 100 \mathrm{~mm}$ Borosilicate glass test tube using a sterile glass Pasteur pipette or glass serological pipette (see Note $^{5}$.

2. Lipids are supplied in chloroform in glass ampoules. Break the cap off the ampoule and transfer lipids, using a glass Pasteur pipette, into a stock glass vial. Gently purge with argon for approximately 10 seconds, to displace any oxygen in the air space, before sealing the glass vial to remove any oxygen $\left(\right.$ see Note $\left.{ }^{6}\right)$. Lipids can be stored in glass vials at $-20^{\circ} \mathrm{C}$ for 6 months.

3. From the stock vial add the volume equivalent to $6.29 \mathrm{mg}$ ( see Note $^{7}$ ) of DOPC to the test tube containing the $2 \mathrm{ml}$ of chloroform. Layer argon over the remaining lipid in the stock glass vial.

4. Proceed to evaporation: clamp the test tube in a universal support clamp. Submerge the bottom two centimetres of the test tube containing the lipid mixture in to a warm, approximately $37^{\circ} \mathrm{C}$, water bath. Gently stream nitrogen over the chloroform-lipid mixture avoiding agitation of the liquid surface. The nitrogen stream can be directed using a glass Pasteur pipette. Stream nitrogen over the mixture until the chloroform evaporates completely (approximately 10 minutes). This creates a lipid film on the bottom of the glass test tube. Once evaporated prevent oxygen from entering the test tube by sealing the opening with parafilm. Repeat steps 1-4 to prepare a $2 \%$ biotinylated DOPC lipid mixture by adding the equivalent of $6.16 \mathrm{mg}$ of DOPC and $0.177 \mathrm{mg}$ of CAP-BIOTIN-PE.

5. Transfer test tubes to a lyophilizer: Poke holes in the parafilm with a hypodermic needle and immediately place inside the vacuum chamber and close the chamber. Turn on the vacuum and lyophilize for a minimum of 90 minutes to remove any residual chloroform.

6. While the samples are in the vacuum, prepare $20 \mathrm{ml}$ of $2 \%$ Octyl $\beta$-D-glucopyranoside and dialysis tubing. The following steps are performed in a laminar flow cabinet using aseptic 
technique. Use $70 \%$ ethanol to clean the flow cabinet workspace and gloves. Periodically clean gloves with $70 \%$ ethanol. Place dialysis tubing beaker in the flow cabinet. Clean dialysis closures (small clips) in $70 \%$ ethanol and set on tissue to dry in the flow cabinet.

\subsection{Solubilisation and sterilization of lipid micelles}

After lyophilisation, lipids are solubilized into detergent by sonication and filter sterilized.

1. Remove the test tubes from the lyophilizer. Remove punctured parafilm and add $2 \mathrm{ml}$ of $2 \%$ Octyl $\beta$-D-glucopyranoside to each lipid containing test tube. Once solubilized this will make $4 \mathrm{mM}$ lipid detergent mixtures. Purge with argon and seal with parafilm.

2. Swirl each tube in a bath sonicator to assist solubilisation of the lipids (approximately $1 \mathrm{~min}$ ). The solution initially becomes turbid before becoming clear. Once clear, remove and place on ice and return to laminar flow cabinet.

3. Filter sterilize the $4 \mathrm{mM}$ lipid detergent mixtures into polypropylene tubes. Purge with argon and immediately seal the tubes (see Note $^{8}$ ). Add $900 \mu 1$ of $2 \%$ Octyl $\beta$-D-glucopyranoside to sterile Eppendorf tubes and add $100 \mu \mathrm{l}$ of appropriate $4 \mathrm{mM}$ lipid detergent mixture to each tube. These will make a $0.4 \mathrm{mM}$ working solutions. Resuspend by pipetting up and down careful not to introduce air bubbles. Purge with argon (see Note $\left.{ }^{9}\right)$

\subsection{Removal of detergent to form liposomes}

1. Transfer $0.4 \mathrm{mM}$ lipid detergent mix to the dialysis tubing using wide tipped sterile disposable Pasteur pipettes. Avoid introducing air bubbles during the transfer. Before sealing the dialysis tubing, sacrifice some lipid detergent mix to remove any air.

2. Place the dialysis tubing in $1 \mathrm{~L}$ of degassed $1 \mathrm{x}$ TBS, add sterile stirrer bar. Seal the conical flask with parafilm and remove any air by purging with argon. Dialyse overnight on a stirrer plate at $4^{\circ} \mathrm{C}$. Ensure that the stirring bar does not form a vortex, which might damage the dialysis membrane. 
3. Perform two further buffer exchanges by transferring the dialysis tubing to fresh conical flask containing $1 \mathrm{~L}$ degassed $1 \mathrm{x}$ TBS, seal with parafilm and purge with argon.

4. In laminar flow hood, cleaned with $70 \% \mathrm{EtOH}$, remove liposomes from the dialysis tubing using wide tipped sterile disposable Pasteur pipettes avoiding air bubbles.

5. Aliquot liposomes into $1.5 \mathrm{ml}$ sterile Eppendorf tubes and layer each tube with argon. Aliquots can be stored at $4^{\circ} \mathrm{C}$ for 3 months ( see Note $^{10}$ )

\subsection{Determine how much recombinant protein is deposited on the bilayer}

Flow cytometry can be used to estimate the number of monobiotinylated recombinant protein molecules incorporated into the upper leaflet of the bilayer. The number of molecules incorporated into the bilayer can be adjusted to mimic the number of molecules endogenously expressed on the surface of a cell. Adjustments can be made by diluting the CAP-BIOTIN-PE containing liposomes with the DOPC liposomes.

1. In a non-tissue culture treated V-bottom 96 well plate add $5 \mu$ l of DOPC liposomes as a negative control or $5 \mu 1$ of CAP-BIOTIN-PE containing liposomes.

2. Add $2.5 \mu \mathrm{l}$ of $5 \mu \mathrm{m}$ diameter hydrophilic silica bead suspension (Bangs Laboratories) to each well. Mix the beads and liposomes together by pulsing on a Vortex mixer.

3. Add $150 \mu \mathrm{l}$ blocking buffer and centrifuge the plate at $600 \mathrm{x} g$ for 2 minutes at room temperature. Remove the supernatant without disturbing the bead pellet. Wash the beads a further 2 times with blocking buffer.

4. Add $50 \mu \mathrm{l}$ of $10 \mu \mathrm{g} / \mathrm{ml}$ streptavidin. Incubate for $20 \mathrm{~min}$ at room temperature with periodic pulsing on a Vortex mixer.

5. Add $150 \mu$ l blocking buffer and centrifuge plate at $600 \mathrm{x} g$ for 2 minutes at room temperature. Remove the supernatant without disturbing the bead pellet. Wash the beads a further 2 times with blocking buffer. 
6. Add $50 \mu \mathrm{l}$ of $10 \mu \mathrm{g} / \mathrm{ml}$ of fluorescently labelled monobiotiylated recombinant protein of known Fluorescent dye: Protein labelling ratio. Incubate for $20 \mathrm{~min}$ at room temperature with periodic pulsing on a Vortex mixer.

7. Add $150 \mu 1$ blocking buffer and centrifuge plate at $600 \times g$ for 2 minutes at room temperature. Remove the supernatant without disturbing the bead pellet. Wash the beads a further 2 times with blocking buffer.

8. Resuspend beads in $150 \mu \mathrm{l}$ blocking buffer and transfer contents of each well to flow cytometer tubes. Add a further $0.3 \mathrm{ml}$ of blocking buffer to each tube and read samples on a flow cytometer followed by Alexa Fluor 488 standard beads labelled with known amounts of Alexa Fluor 488 (Bangs Laboratories).

9. Generate a linear standard curve by plotting the number of Alexa Fluor 488 molecules on the $x$ axis against the median fluorescence intensity (MFI) on the $y$ axis.

10. Use the equation of the line defined in step 9 to determine the number of fluorescent dye molecules associated with the bilayer coated beads.

11. Use the equation below to calculate the density of monobiotinylated molecules (molecues $/ \mu \mathrm{m}^{2}$ ) tethered to the bilayers using the Fluorescent dye: Protein labelling ratio and the surface area of the silica beads $\left(78.54 \mu \mathrm{m}^{2}\right.$ for $5 \mu \mathrm{m}$ diameter beads)

(Sample MFI) / (Fluorescent dye: Protein ratio) $=$ density molecules $/ \mu \mathrm{m}^{2}$

Bead surface area

\subsection{Preparation of glass coverslips for forming artificial lipid bilayers}

1. A lipid bilayer will spontaneously form when liposomes fuse to ultraclean glass. Remove any visible debris from $40 \mathrm{~mm}$ diameter glass coverslips with a tissue. Clamp at one edge of the coverslip with Nalge Nunc polypropylene scissor type forceps.

2. Submerge coverslips in freshly prepared piranha solution while remaining clamped with the forceps, leaving them submerged for 15 to 20 mins (see Note ${ }^{11}$ ). 
3. Carefully remove the forceps from the piranha solution and extensively rinse the coverslip with ultrapure water.

4. While still holding the coverslip with forceps, dry using a vacuum line attached to a clean pipet tip (see Note $^{12}$ ). Keep the coverslip clamped in the forcep until ready to use.

\subsection{Forming artificial lipid bilayers in a flow cell}

To form artificial lipid bilayers we use a Bioptechs FCS2 chamber with integrated heating.

1. The upper half of the chamber, which contains the perfusion tubes, is placed on a flat surface with the perfusion tubes pointing upwards. Position the circular gasket with the holes over the perfusion tubes. Align the holes and position the microaqueduct slide over the gasket with the fluidic channels facing upwards (see Note ${ }^{13}$ ). Layer the $0.25 \mathrm{~mm}$ gasket with rectangular cut-out over the microaqueduct slide.

2. Position up to $5 \times 1 \mu 1$ spots of liposome suspension on the surface of exposed microaqueduct slide surface (Fig 1). These liposome droplets will form small domes.

3. In a single motion, position a dry piranha treated $40 \mathrm{~mm}$ coverslip over the gasket with rectangular cut-out. A contact will be made between the top of the droplet and the coverslip. Immediately clamp the chamber together with the self-locking base. Mark the position of the bilayers with permanent marker pen and leave to stand for 20 minutes at room temperature.

4. Meanwhile prepare the tubing which will permit the addition of 1x PBS, blocking buffer and components which will be tethered to the bilayer. Place an unprimed piece of tubing with a two-way stopcock in the open position to the exit perfusion tube of the chamber. This feeds into a waste beaker.

5. A three-way stopcock enables components to be added to the microfluidic chamber without the addition of air bubbles ( see Note ${ }^{14}$ ). Prior to connecting the tubing with the three-way stopcock to the FCS2 chamber prime the tubing, stopcock and the dead volume in the short length of tubing between the stopcock and the chamber with 1x PBS to remove any air trapped in the tubing, (the setup up can be viewed in Fig 2). Connect the tubing to the 
chamber and in single motion, gently but firmly, push through $5 \mathrm{ml}$ of 1x PBS while visually confirming no air bubbles pass over the bilayers. Close off the chamber.

6. Block the bilayers with $1 \mathrm{ml}$ of blocking buffer. Take up the blocking buffer into a $1 \mathrm{ml}$ syringe. Remove any air bubbles by gently tapping the syringe. Expel some blocking buffer to produce a convex meniscus. Prime the stopcock port with 1x PBS and leave a convex meniscus. Attached the syringe containing blocking buffer by bringing the convex meniscus of the syringe to the convex meniscus of the stopcock. This method will prevent the introduction of air to the system. Change the stopcock position to close off the $20 \mathrm{ml} 1 \mathrm{x}$ PBS containing syringe. Inject the blocking buffer into the chamber (see Note ${ }^{15}$ ). Close off the chamber and incubate for 20 minutes at room temperature. When incubating use the 2 and 3 way stopcocks to seal the chamber.

7. Unseal the chamber and gently remove unbound blocking buffer by passing approximately $5 \mathrm{ml}$ of $1 \mathrm{x}$ PBS through the chamber. Using the same method described in step 6 add 10 $\mu \mathrm{g} / \mathrm{ml}$ streptavidin. Incubate for 20 minutes at room temperature. Remove unbound streptavidin by passing approximately $5 \mathrm{ml}$ of $1 \mathrm{x}$ PBS through the chamber. Add monobiotinylated platelet ligand and incubate 20 minutes at room temperature. Remove unbound monobiotinylated ligand by passing approximately $5 \mathrm{ml}$ of $1 \mathrm{x}$ PBS through the chamber.

\subsection{Imaging bilayers}

The flow chamber can be mounted onto an inverted microscope and the dynamics of fluorescently labelled ligands can be monitored using total internal reflection fluorescence microscopy and other microscopy techniques (Fig 3). Prior to the addition of platelets into the chamber the mobility of the bilayer must be confirmed. This is achieved by fluorescence recovery after photobleaching (FRAP) where an area of fluorescent molecules are bleached and the movement of non-bleached molecules into the bleached area can be followed (Fig 4). 
Once the mobility of the bilayer has been confirmed, platelets, at $2-3 \times 10^{7}$ platelets $/ \mathrm{ml}$ can be introduced into the chamber using the three-way stopcock using the technique described above to avoid the introduction of air bubbles into the chamber.

\section{Figures}

Figure 1: Layout of the $1 \mu \mathrm{l}$ liposome droplets on the microaqueduct slide. Up to 5 droplets can be positioned at one time and can include a mixture of DOPC (negative control) and CAP-BIOTIN-PE containing liposomes.

Figure 2: Layout of the perfusion tubing including the positioning of the two-way and three-way stopcocks.

Figure 3: Representative images of platelet mediated clustering of artificial lipid bilayer tethered platelet ligands. A single platelet is clustering a fluorescently labelled monobiotinylated activating antibody tethered to the upper leaflet of an artificial lipid bilayer. Scale bar $2 \mu \mathrm{m}$.

Figure 4: Prior to the addition of platelets into the chamber the lateral mobility of the bilayer must be confirmed. This is achieved by fluorescence recovery after photobleaching (FRAP). Here, two areas of fluorescent molecules are bleached and the movement of non-bleached molecules into the bleached area followed.

\section{References}

1. Fooksman DR, Vardhana S, Vasiliver-Shamis G, Liese J, Blair DA, Waite J, Sacristan C, Victora GD, Zanin-Zhorov A, Dustin ML (2010) Functional anatomy of T cell activation and synapse formation. Annu Rev Immunol 28:79-105. doi:10.1146/annurev-immunol-030409-101308

2. Groves JT, Dustin ML (2003) Supported planar bilayers in studies on immune cell adhesion and communication. J Immunol Methods 278 (1-2):19-32 
3. Fleire SJ, Batista FD (2009) Studying cell-to-cell interactions: an easy method of tethering ligands on artificial membranes. Methods in molecular biology (Clifton, NJ) 462:145-154. doi:10.1007/9781-60327-115-8_9

4. Pollitt AY, Poulter NS, Gitz E, Navarro-Nunez L, Wang YJ, Hughes CE, Thomas SG, Nieswandt B, Douglas MR, Owen DM, Jackson DG, Dustin ML, Watson SP (2014) Syk and Src family kinases regulate C-type lectin receptor 2 (CLEC-2)-mediated clustering of podoplanin and platelet adhesion to lymphatic endothelial cells. The Journal of biological chemistry 289 (52):35695-35710.

doi:10.1074/jbc.M114.584284

5. Dustin ML, Starr T, Varma R, Thomas VK (2007) Supported planar bilayers for study of the immunological synapse. Current protocols in immunology Chapter 18:Unit 18.13. doi:10.1002/0471142735.im1813s76

6. Watson SP, Herbert JM, Pollitt AY (2010) GPVI and CLEC-2 in hemostasis and vascular integrity. Journal of thrombosis and haemostasis : JTH 8 (7):1456-1467. doi:10.1111/j.1538-7836.2010.03875.x

7. Nye JA, Groves JT (2008) Kinetic control of histidine-tagged protein surface density on supported lipid bilayers. Langmuir : the ACS journal of surfaces and colloids 24 (8):4145-4149. doi:10.1021/la703788h

8. Valvo S, Mayya V, Seraia E, Afrose J, Novak-Kotzer H, Ebner D, Dustin ML (2017)

Comprehensive Analysis of Immunological Synapse Phenotypes Using Supported Lipid Bilayers. Methods in molecular biology (Clifton, NJ) 1584:423-441. doi:10.1007/978-1-4939-6881-7_26

9. Schutz GJ, Huppa JB (2017) Forster Resonance Energy Transfer to Study TCR-pMHC Interactions in the Immunological Synapse. Methods in molecular biology (Clifton, NJ) 1584:207-229. doi:10.1007/978-1-4939-6881-7_14

10. Su X, Ditlev JA, Rosen MK, Vale RD (2017) Reconstitution of TCR Signaling Using Supported Lipid Bilayers. Methods in molecular biology (Clifton, NJ) 1584:65-76. doi:10.1007/978-1-49396881-7_5 
${ }^{1}$ The buffers are degassed to deoxygenate them. This step protects the liposomes from oxidation during long-term storage.

${ }^{2}$ Wear a full lab coat and chemical-resistant apron, chemical-resistant gloves. Preparation of piranha solution must occur in a fume hood. It is expected that this solution will heat up to over $100^{\circ} \mathrm{C}$ and begins to produce small bubbles. Measure components carefully as making the solution greater than $50 \%(\mathrm{v} / \mathrm{v})$ with respect to the $30 \%(\mathrm{w} / \mathrm{w})$ hydrogen peroxide $\left(\mathrm{H}_{2} \mathrm{O}_{2}\right)$ can result in a more violent reaction and possible explosion. Always add the hydrogen peroxide to the sulfuric acid slowly and in this order. Never mix piranha solution with organic compounds as this will results in a violent reaction. Consult local standard operating procedures regarding the disposal of piranha solution waste. Some institutions permit cooled piranha waste to be disposed into the drains with copious amounts of water.

${ }^{3}$ The ability to purchase biotinylated lipids allows for the incorporation of biotinylated proteins into the upper leaflet of the bilayer using streptavidin to bridge the two. It is important that proteins have a single biotin per molecule. This can be achieved by biotinylating recombinant proteins chemically using conditions where the majority of molecules have a single biotin. Alternatively proteins can be biotinylated in a directed manner using a BirA tag. If molecules have multiple biotins the protein will crosslink and aggregate in the bilayer and will become immobile.

${ }^{4}$ The protocol described here employs a dialysis method to prepare liposomes. This method is the gentlest method for making proteoliposomes, liposomes in which one or more proteins have been inserted. Where proteoliposomes are not being employed alternative methods can also be employed to prepare liposomes. These include extrusion, sonication and freeze-thaw methods [8-10].

${ }^{5}$ All work involving Chloroform must occur in a fume hood. Chloroform is a solvent and therefore the interaction of Chloroform and plastics should be avoided.

${ }^{6}$ Purging with argon removes any oxygen preventing oxidation of the lipids.

${ }^{7}$ Different concentrations of lipids are available. For example if a $10 \mathrm{mg} / \mathrm{ml} \mathrm{stock}$ solution of DOPC is purchased $629 \mu \mathrm{l}$ of DOPC in chloroform will be needed. To prepare a $2 \%$ CAPBIOTIN-PE:DOPC lipid mix $616 \mu \mathrm{l}$ of $10 \mathrm{mg} / \mathrm{ml}$ DOPC and $17.7 \mu \mathrm{l}$ of $10 \mathrm{mg} / \mathrm{ml}$ CAP-BIOTINPE are added.

8 Avoid pushing too strongly on the filter as too much pressure will cause air bubbles to form.

${ }^{9}$ When purging oxygen from Eppendorf tubes using argon is it important that the argon flow is reduced. If the flow is too high the liquid will be displaced and lost.

${ }^{10}$ To test for sterility add a small volume of each lipid to a petri dish with media and store in incubator at $37^{\circ} \mathrm{C}$. Monitor for any media contamination over subsequent days. 
${ }^{11}$ One $100 \mathrm{ml}$ glass beaker can hold a maximum of 4 forcep held coverslips. It is advisable to prepare extra coverslips to mitigate against coverslip breakages.

12 There should be no visible residue or particles remaining on the glass. After drying, coverslips should be used within a day and protected from dust if exposed to air for any length of time. Dust may be blown off with a compressed-air duster.

${ }^{13}$ Ensure that the microaqueduct slide is well fitted over the perfusion tubes. If the holes are not aligned the microaqueduct slide will crack when the chamber is clamped together.

${ }^{14}$ Air bubbles will destroy the bilayer rendering them unusable.

${ }^{15}$ When introducing buffers to the chamber ensure that the exit two-way stopcock is open. When not passing components through the chamber then turn this stopcock to the off position. 